Voix et Images

voixetimages

\title{
Trois livres en équilibre sur une époque
}

\section{François Paré}

Volume 35, numéro 1 (103), automne 2009

Herménégilde Chiasson

URI : https://id.erudit.org/iderudit/038574ar

DOI : https://doi.org/10.7202/038574ar

Aller au sommaire du numéro

\section{Éditeur(s)}

Université du Québec à Montréal

\section{ISSN}

0318-9201 (imprimé)

1705-933X (numérique)

Découvrir la revue

Citer ce compte rendu

Paré, F. (2009). Compte rendu de [Trois livres en équilibre sur une époque].

Voix et Images, 35(1), 115-119. https://doi.org/10.7202/038574ar d'utilisation que vous pouvez consulter en ligne.

https://apropos.erudit.org/fr/usagers/politique-dutilisation/ 


\section{E S S A I S / ÉT U D E S}

Trois livres en équilibre sur une époque

$++$

FRANÇOIS PARÉ

Université de Waterloo

Dans un curieux passage de Nombres, Philippe Sollers fait appel à une métaphore spatiale pour parler du rôle que chacun joue devant l'histoire: «il y avait à choisir entre l'est et l'ouest, et c'était comme si l'on avait à se décider pour un passé à faux visage de futur ou pour un futur encore figuré au passé ${ }^{1}$...» À l'est, le romancier dit apercevoir "la force invisible des mutations complètes», tandis que, tourné vers l'occident, son regard se laisse plutôt émouvoir par «la terre parlante et armée et comme se réveillant d'un sommeil calculé» (37). Entre ces deux pôles concurrents qui ne cessent d'évoluer, le sujet, à mi-chemin entre le présent et l'avenir, n'arrive plus à faire la part du «thêâtre » qui continue de se jouer devant lui et le plus souvent de lui. Si l'opposition est-ouest ne s'applique plus de la même façon, quarante ans après la parution du roman de Sollers, la rupture problématique qu'elle laisse entendre doit bien encore fasciner notre époque. La littérature et les discours multiples auxquels elle donne lieu (dont celui-ci!) cherchent toujours à ranger les éléments épars de la culture, à la fois pour confirmer l'archivage nécessaire du passé et souligner la menace que poserait néanmoins son total oubli. En littérature québécoise, de nombreux écrits récents ont voulu tenir compte d'un ensemble complexe de notions qui ont déterminé le sens à donner à l'histoire, avec en premier lieu le puissant imaginaire de la révolution tranquille. «Parcour[ant] les ruines de ce passé s'obstinant à ne pas passer» (p. 70), les trois ouvrages dont il est question dans cette chronique soulèvent certaines conceptions figées liées à l'historiographie littéraire récente du Québec et évoquent ainsi un jeu d'équilibre à la fois précaire et lumineux.

Dans les premières pages de son excellent essai sur la réception critique de trois œuvres romanesques majeures des années 1960, Martine-Emmanuelle Lapointe nous met en garde contre toute lecture abusive de l'histoire littéraire: «Relire une histoire, tenter d'identifier ses auteurs et ses acteurs, témoigner de la diversité des

$$
+++
$$

1 Philippe Sollers, Nombres, Paris, Éditions du Seuil, coll. «Tel Quel», 1968, p. 36. 
voix et des accents qui la composent, une telle entreprise comporte des risques. Comment en effet pourrais-je prétendre à l'exhaustivité et à l'objectivité alors que ma propre parole est mise en jeu, précaire, fragile, datée, historique ${ }^{2}$ ? » Il s'instaure alors un dialogue, parfois ironique, parfois simplement étonné, entre le présent et le passé, car cette histoire est archi-connue et nous lui sommes malgré tout redevables. Le retour que nous propose Lapointe sur la réception critique du Libraire de Gérard Bessette, de Prochain épisode d'Hubert Aquin et de L'avalée des avalés de Réjean Ducharme nous entraîne bien au-delà du simple compte rendu de lecture, puisque nous sommes au cœur même de ce "grand récit» de la révolution tranquille sur lequel se fonde encore la modernité québécoise. Par son travail sur les «figures du recommencement» (40), l'essayiste cherche à comprendre les procédures de sélection, de classement et de censure qui ont contribué à marquer la fin de la culture canadiennefrançaise et à produire la toute première histoire d'une littérature appelée québécoise.

Dès sa parution en France et au Québec en 1960, Le libraire de Gérard Bessette est interprété comme une histoire de résistance, «transformant un être asocial, résolu à l'aphasie et à l'inertie, en un héros, liminaire le plus souvent sans doute, mais tout de même tourné vers l'action» (84). L'unanimité critique semble se former assez rapidement autour du personnage de Jodoin que chacun voit comme le témoin de la censure duplessiste et l'agent paradoxal d'une transformation sociale à venir. C'est l'interdiction du livre qui frappe surtout l'imagination, comme si le silence ne s'imposait que de l'extérieur, mis en œuvre par une autorité honnie. Pourtant, les personnages de Bessette sont hantés par leur démission devant le langage et leur incapacité à s'exprimer. À la lecture conventionnelle du Libraire, Martine-Emanuelle Lapointe oppose une analyse qui, sans renoncer à l'histoire politique du roman, serait attentive à d'autres questions telles que la communication faussée, l'ennui et l'épuisement des valeurs morales. La médiocrité du personnage de Jodoin naîtrait du refus de la «Parole» - concept emblématique, s'il en est un, de la révolution tranquille! - et de ce mutisme désabusé qui le place résolument en retrait du monde.

Si Prochain épisode d'Hubert Aquin est considéré, lors de sa publication en 1965, comme un reflet des «nouvelles» formes romanesques, la plupart des critiques lui confèrent néanmoins une valeur politique. La destinée du narrateur errant et sans avenir, mis en scène par Aquin, s'apparente aux yeux de tous à l'histoire collective du peuple québécois. Le héros aquinien est déchiré entre la démobilisation et l'action. Son angoisse n'est-elle pas celle de toute la collectivité devant les choix qui s'imposent à elle et qui constituent sa modernité particulière? Martine-Emmanuelle Lapointe offre une analyse très fine des textes fondamentaux d'Hubert Aquin sur l'exercice de la littérature en situation dominée. Certes, dans «Littérature et aliénation» et dans "Profession: écrivain», Aquin refusait à l'artiste toute pertinence et surtout toute efficacité. Mais Lapointe fait bien voir que " [s]i l'art est ainsi discrédité, c'est parce qu'il enferme l'homme dans une fonction et le condamne au respect d'un certain ordre social» (159). Prochain épisode contient donc, beaucoup plus que

2 Martine-Emmanuelle Lapointe, Emblèmes d'une littérature. Le libraire, Prochain épisode et L'avalée des avalés, Montréal, Fides, coll. «Nouvelles études québécoises», 2008, p. 33. 
d'autres romans de la période, le ferment d'une transformation de la société par une pratique concertée du désordre.

Paru un an après le roman d'Aquin, L'avalée des avalés de Réjean Ducharme connaît un succès réel sur le marché français et suscite dès lors, comme on peut s'y attendre, une bonne dose de méfiance au Québec. Toutefois, l'œuvre frappe la critique de l'époque par sa vibrante intertextualité et par l'indépendance ludique qu'elle professe. Cependant, "[a]u même titre que Le libraire et Prochain épisode, L'avalée des avalés est rapidement soutenu et absorbé par le discours institutionnel, tant et si bien qu'il semble impossible de tracer une frontière nette entre sa première réception et son entrée dans les textes de synthèse et les histoires littéraires » (264). Par le mystère qu'il crée, Ducharme devient l'image même d'une modernité dont on apprécie la polyvalence et la capacité d'intégrer toutes les différences. Le livre de Martine-Emmanuelle Lapointe démontre ainsi de façon remarquable «le caractère construit [de] nos récits mémoriels» (317). Mais cette construction ne nous est visible que par reflets et fragments. Le passé reste la face cachée du présent.

Le livre se lit d'un trait, chose rare, il faut bien le dire, dans le cadre d'une chronique sur l'essai critique... À certains moments, nous avons l'impression d'être en plein polar: mensonges, cachotteries, abus d'influence, jalousies et rivalités se succèdent, alors que les écrivains et leurs mécènes se voient forcés de révéler leurs petits secrets et leurs contradictions. La juxtaposition de textes brefs et de magnifiques portraits photographiques fascine le regard. Toute la «famille» littéraire y figure; tous les grands moments y sont représentés. Troisième volet d'une grande étude de l'histoire des prix et des subventions littéraires au Québec, le Dictionnaire-album du mécénat $d^{\prime}$ Etat de Robert Yergeau ${ }^{3}$ retrace, dans une forme tout autre, les liens étroits qui se sont tissés depuis le début du $\mathrm{XX}^{\mathrm{e}}$ siècle entre les écrivains québécois et les organismes subventionnaires nationaux. Pour mener son enquête, le chercheur a consulté des centaines de documents d'archives, certains du domaine public et d'autres obtenus des gouvernements en faisant appel au droit d'accès à l'information. Cet album étonnant, dans lequel les commentaires parfois caustiques de Yergeau alternent avec de très belles photos d'époque, se concentre sur un assez grand nombre d'acteurs connus et moins connus des milieux littéraires et subventionnaires. Certains personnages, tels Victor-Lévy Beaulieu, Jacques Godbout, Gaston Miron, Nicole Brossard ou Naïm Kattan, font l'objet d'un éclairage plus soutenu et plus décapant. Certaines pages, particulièrement bien documentées, touchent les grands mouvements littéraires qui ont marqué le tournant des années 1960 au Québec: Parti pris, Les Herbes Rouges, Liberté. Yergeau ne ménage aucunement les deux grands bailleurs de fonds que sont le ministère des Affaires culturelles du Québec et le Conseil des arts du Canada.

Ce qui frappe à la lecture de cet ouvrage, c'est la fragilité extrême des instances littéraires concurrentes. On se rend compte que nombre d'écrivains ont vécu

$$
+++
$$

3 Robert Yergeau, Dictionnaire-album du mécénat d'État, Ottawa, Le Nordir, coll. «Roger-Bernard», 2009, 205 p. 
et continuent de vivre dans le plus grand dénuement matériel et dans la dépendance quasi totale à l'égard des gouvernements. Il est clair aussi que la littérature obtient la part congrue des déboursés gouvernementaux en matière d'art, et les subventions reçues par les auteurs sont dérisoires. C'est pourquoi, incapables de payer les comptes, les écrivains hésitent rarement, par exemple, à mettre de côté leurs scrupules politiques pour pouvoir se prévaloir de la manne du Conseil des arts du Canada. Robert Yergeau choisit ses exemples avec soin. Ainsi, «[p]oète de la marginalité la plus intransigeante» (176), Denis Vanier a tout de même reçu douze bourses du Conseil des arts du Canada et presque autant de son équivalent québécois. Citant les lettres d'appui rédigées par des confrères écrivains, Yergeau montre comment le milieu littéraire a camouflé les enjeux de l'œuvre de Vanier tout en prenant soin de maintenir l'image du "poète maudit» que celui-ci aimait projeter. «Maudit par qui ? " se demande le critique: "Denis Vanier a pu recevoir une telle manne étatique parce que son œuvre a été prise en charge par un univers de célébrants et de croyants dans une trajectoire institutionnelle qui l'a conduit de la marge au centre, fût-ce un centre marginalisé, malgré l'apparent paradoxe d'une telle formule.» (176) Les exemples de ce type de travestissements idéologiques abondent, selon Yergeau, surtout si l'on tient compte des nombreux cas d'écrivains souverainistes qui, tout en décriant publiquement le Conseil des arts du Canada, profitaient secrètement de ses largesses. Le critique n'est pas tendre devant ces impostures assez prévisibles, et son commentaire est souvent empreint de cynisme et d'exaspération.

Outre les superbes photographies, et en particulier le fascinant montage réalisé par Christian Quesnel sur la jaquette du livre, la part la plus originale de cet ouvrage touche sans doute les pages consacrées au rôle déterminant de certains fonctionnaires au sein des organismes subventionnaires et des jurys chargés de l'étude des dossiers de demandes de bourses. De nombreuses subventions ont été octroyées sans qu'aucune évaluation ne soit faite par les membres d'un jury, alors que d'autres, pourtant recommandées, ont été refusées en dernière instance par les ministères. À Ottawa, dès les premières heures du Conseil des arts du Canada, les intercessions d'Andrée Paradis, alors directrice de la revue Vie des arts, en faveur de certains écrivains, tel Gaston Miron, leur ont permis de déjouer les procédures de sélection. Par ailleurs, ses réserves à l'égard de l'équipe de la revue Liberté ont eu un impact négatif sur son financement. À la tête du Service des lettres et de l'édition du Conseil des arts du Canada de 1967 à 1990, Naïm Kattan a occupé une position primordiale sur une très longue période. Il est clair, selon Yergeau, que Kattan régnait sur toutes les opérations du Conseil et donnait son autorisation finale à la moindre subvention. Peu d'écrivains québécois, à l'exception de Gérard Bessette et d'Adrien Thério, ont osé remettre en cause le pouvoir de légitimation que Kattan détenait. Une atmosphère encore moins amène, semble-t-il, régnait au bureau de Clément Saint-Germain, le responsable des programmes de subventions au ministère des Affaires culturelles à Québec; et les dissidents se faisaient rares. Le Dictionnairealbum du mécénat d'État poursuit donc l'enquête admirable entreprise par Robert Yergeau, il y a une dizaine d'années. Il fait voir sans aucun scrupule les «en-dessous » plus ou moins admirables de la littérature québécoise au moment où le travail des écrivains et du milieu littéraire dans son ensemble s'institutionnalisait. 
Un recueil d'articles sur Jacques Brault forme le troisième volet de ce triptyque posé comme en équilibre sur une époque. Sous la direction de François Hébert et de Nathalie Watteyne, Précarités de Brault est issu d'un colloque consacré à cet auteur en octobre $2004^{4}$. L'ouvrage se veut à la fois un hommage au professeur de littérature aujourd'hui à la retraite et une étude de certains aspects de son œuvre poétique, romanesque et essayistique. Modeste, fervente et mélancolique, l'écriture de Brault reste très proche de la conscience actuelle. Quelque cinquante ans après la parution des premières œuvres, elle nous accompagne toujours, tant les stratégies et les postures de l'écrivain nous semblent naturelles. Les auteurs du présent collectif ne cherchent pas, du reste, à rompre avec cette amitié fusionnelle: ils veulent, disentils, faire un bout de chemin avec Brault, le «saluer» en «faisant écho à sa voix» (18).

Il est souvent question de l'espace dans les textes de ce collectif. Ainsi, voulant parler de la langue chez Brault, Karim Larose finit par souligner l'importance de la rue, «lieu où le temps s'expose en exposant [...]. Au coin de la rue, les odeurs prennent à la gorge, les accents refont surface, la langue maternelle atteint avec force le passant distrait» (41). Larose fait voir que le visage enjoué de la ville cache, chez le poète, la poussière des usines et la «blessure fidèle » de ceux qui chaque jour sont prisonniers de son rythme. La langue ne se laisse pas saisir sans ses attaches dans cette quotidienneté à la fois solitaire et bruyante. À l'inverse, nombreux sont les textes qui mettent l'accent sur l'importance du silence dans l'œuvre de Brault. Pour Nathalie Watteyne, cette référence, inspirée peut-être d'une lecture de Saint-Denys Garneau, constitue un au-delà de la parole, une sorte de transcendance qui serait blottie au cœur même des mots et qui nous aiderait à «surmonter les ambivalences » (32) de l'existence.

L'ouvrage présente également certains témoignages de contemporains, tels Jean-Pierre Issenhuth, Gilles Marcotte et Robert Melançon qui, tous trois, fournissent de nombreux détails sur la composition et l'évolution des différents recueils. Ces réflexions personnelles sont marquées par le même esprit de patience qui semble entourer l'écriture de Brault. Jacinthe Martel s'intéresse au travail conjoint de Jacques Brault et de Robert Melançon dans Au petit matin et Au fond du jardin. Son étude minutieuse de la genèse de certains extraits révèle l'extraordinaire érudition du poète. L'intertextualité se présente chez lui comme une «mémoire intimiste» qui se fait «doucement entendre» (205). C'est ainsi que la «figure du déserté», celle de l'écrivain hanté par la mort, devient à nouveau habitée. Elle se tient au bord de la mer, comme l'imagine Mélanie Beauchemin dans son bel article sur Agonie: elle est tendue vers la rencontre, vers le multiple. Ce sont de telles images qui rendent l'œuvre de Jacques Brault si étonnamment présente, encore maintenant, comme si, à elle seule, elle pouvait recoudre tous les tissus déchirés du passé et aplanir tous les doutes sur la légitimité du présent.

$$
+++
$$

4 François Hébert et Nathalie Watteyne (dir.), Précarités de Brault, Québec, Nota bene, coll. «Convergences», 2008, 215 p. 